\title{
MULTIDRUG RESISTANT Pantoea agglomerans IN A PATIENT WITH SEPTIC ARTHRITIS- A RARE REPORT FROM INDIA
}

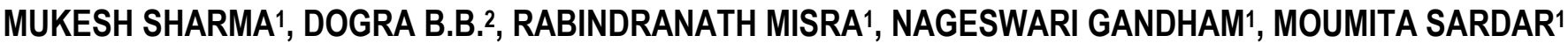 \\ AND SAVITA JADHAV ${ }^{*}$
}

1Department of Microbiology, Pad. Dr. D. Y. Patil Medical College, Pimpri, Pune-411 018, MS, India.

2Department of Surgery, Pad. Dr. D.Y. Patil Medical College, Pimpri, Pune-411 018, MS, India.

*Corresponding Author: Email- patilsv78@gmail.com

Received: July 11, 2012; Accepted: July 19, 2012

\begin{abstract}
-
Introduction- Pantoea agglomerans ( $P$. agglomerans) is gram negative bacterium that belongs to the family Enterobacteriaceae. It is an opportunistic pathogen in the immunocompromised, causing wound infections, bacteremia, and urinary tract infections. This species is currently listed as a Biosafety level 2 (BL2) organism due to clinical reports as an opportunistic human pathogen. Septic arthritis or synovitis is usually common, clinical manifestations caused by $P$. agglomerans and often correlated with a predisposing factor i.e. immunodeficiency (diabetes mellitus, malignancies, extremes of age) or use of central catheter. This is first described case of septic arthritis following a road side injury.

Case Report- In May 2012, A 40 year old male was admitted to the hospital with pain in left leg since last three months and swelling in his left knee for 4 days which was insidious in onset. On physical examination he was a febrile. His left knee was warm, swollen and tender. Three days later he became febrile and swelling gradually progressed resulting in cellulites. Ampicillin and Gentamicin were initiated/ started empirically. Later on an ill defined ulcer with irregular margin developed associated with purulent discharge. Patient was diagnosed as septic arthritis. Arthrocentesis was done and aspiration of synovial fluid was sent for culture and sensitivity to microbiology department and culture growth was phenotypically identified as $P$. agglomerans. The final identification of the bacteria by the Vitec 2 Systems (bioMerieux, France) was $P$. agglomerans. with multidrug resistant pattern in in-vitro testing .
\end{abstract}

Key words- Pantoea agglomerans, septic arthritis, extended spectrum $\beta$-lactamases

Citation: Mukesh Sharma, et al. (2012) Multidrug Resistant Pantoea agglomerans in a Patient with Septic Arthritis-a Rare Report from India. International Journal of Microbiology Research, ISSN: 0975-5276 \& E-ISSN: 0975-9174, Volume 4, Issue 6, pp.-263-265.

Copyright: Copyright@2012 Mukesh Sharma, et al. This is an open-access article distributed under the terms of the Creative Commons Attribution License, which permits unrestricted use, distribution and reproduction in any medium, provided the original author and source are credited.

\section{Introduction}

Pantoea agglomerans ( $P$. agglomerans) is gram negative bacterium that belongs to the family Enterobacteriaceae. It is an opportunistic pathogen in the immunocompromised, causing wound infections, bacteremia, and urinary tract infections [1]. P. agglomerans, formerly named Enterobacter agglomerans is less often implicated in infection than Enterobacter aerogenes and Enterobacter cloacae and usually complicates debilitating illnesses. $P$. agglomerans strains are among the most promising biocontrol agents for a variety of bacterial and fungal plant diseases, particularly fire blight of apple and pear. This species is currently listed as a Biosafety level 2 (BL2) organism due to clinical reports as an opportunistic human pathogen [2-4]. Septic arthritis or synovitis is usually common, clinical manifestation caused by $P$. agglomerans and often correlated with a predisposing factor i.e. immunodeficiency (diabetes mellitus, malignancies, extremes of age) or use of central catheter [2-5]. This is first described case of septic arthritis following a road side injury in India.

\section{Case Report}

In May 2012, A 40 year old male was admitted to this hospital with pain in left leg since last three months and swelling in his left knee 
for 4 days which was insidious in onset. Patient was febrile and his left knee was warm, swollen and tender.

The patient gave a history of road traffic accident and injury one year back and for which he was operated in another hospital. Following operation there was foul smelling discharge from wound on knee joint approximately $4 \times 4 \mathrm{~cm}$ for which he was advised cefotaxime at the time of discharge.

The patient did not gave any history of diabetes mellitus, hypertension, HIV, tuberculosis or bronchial asthma. He was treated symptomatically with intramuscular NSAIDs. Three days later he became febrile and swelling of the knee gradually progressed superficially resulting in cellulitis of supra patellar region. He was put on broad spectrum antibiotics empirically. Later on an ill defined ulcer with irregular margin developed on lateral side of left knee associated with purulent discharge [Fig. 1].

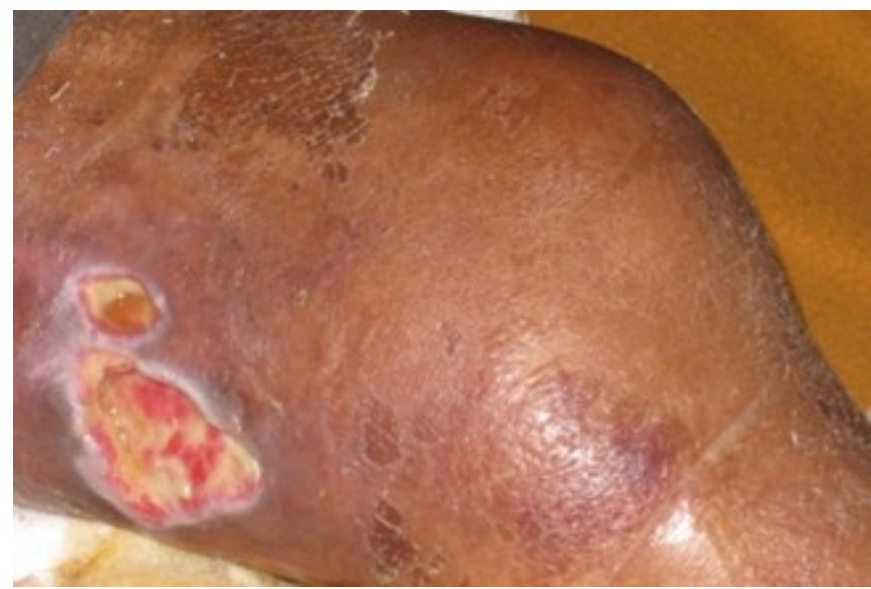

Fig. 1- Wound with Purulent Discharge

At the time of admission routine investigations revealed leukocytosis of $23000 / \mathrm{cmm}$ with $80 \%$ neutrophils. Blood Erythrocyte sedimentation rate (ERS) was $30 \mathrm{~mm} / \mathrm{h}$. Blood sugar fasting was 215 $\mathrm{mg} / \mathrm{dl}$ and post prandial level was $290 \mathrm{mg} / \mathrm{dl}$. He was diagnosed to have diabetes mellitus and insulin was started. HIV was nonreactive. X-ray of left knee showed only soft tissue swelling and no foreign body was detected in X-ray. USG of left knee showed sub cutaneous edema around joint. Patient was diagnosed to be case of septic arthritis left knee and managed conservatively. Subsequently he developed ulceration on left supra patellar region associated with purulent discharge. Pus sample was collected from suppurative wound and send to microbiology department for culture and sensitivity but there was no growth. Arthrocentesis of left knee was carried out and synovial fluid was aspirated and sent for culture and sensitivity to microbiology department.

\section{Lab Diagnosis}

Gross examination of synovial fluid was opaque pale yellow coloured synovial fluid. Direct microscopy of Gram stained of fluid showed plenty of pus cells with intracellular Gram negative bacilli (GNB) [Fig. 2]. Sample was inoculated on Blood agar (BA) and MacConkey agar (MA) and incubated overnight at $37^{\circ} \mathrm{C}$ aerobically in incubator. Gram stain of growth in liquid broth revealed sausage shaped aggregations or symplasmata [Fig. 3] ( appearing as chains of oblong cells). After overnight incubation BA showed pale yellow coloured convex colonies of 2-3 mm diameter in size, non-hemolytic. On MA lactose fermenting, punctuate, umbilicated, convex, smooth glistening colonies were grown [Fig.4]. The Gram staining of colony of organism showed GNB, which were motile, catalase positive and oxidase negative with fermenting sugars and phenotypically identified as $P$. agglomerans. The final identification of the bacteria by the Vitec 2 Systems (bioMerieux, France) was $P$. agglomerans.

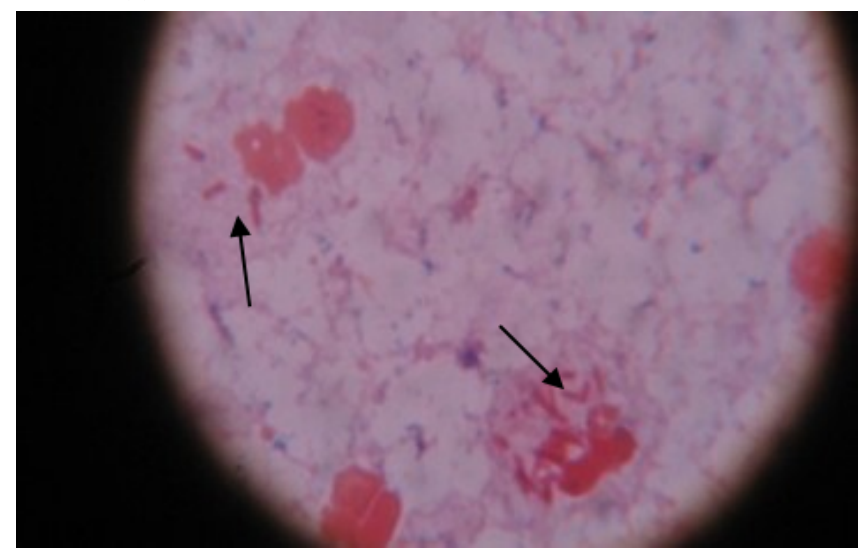

Fig. 2- Grams staining of synovial fluid showing intracellular gram negative bacilli.

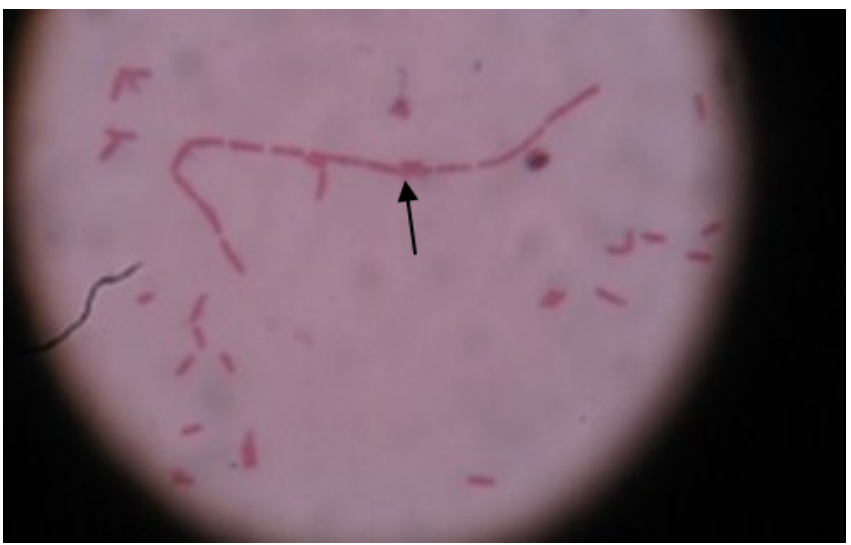

Fig. 3- Grams staining of Liquid Media showing Symplasmata

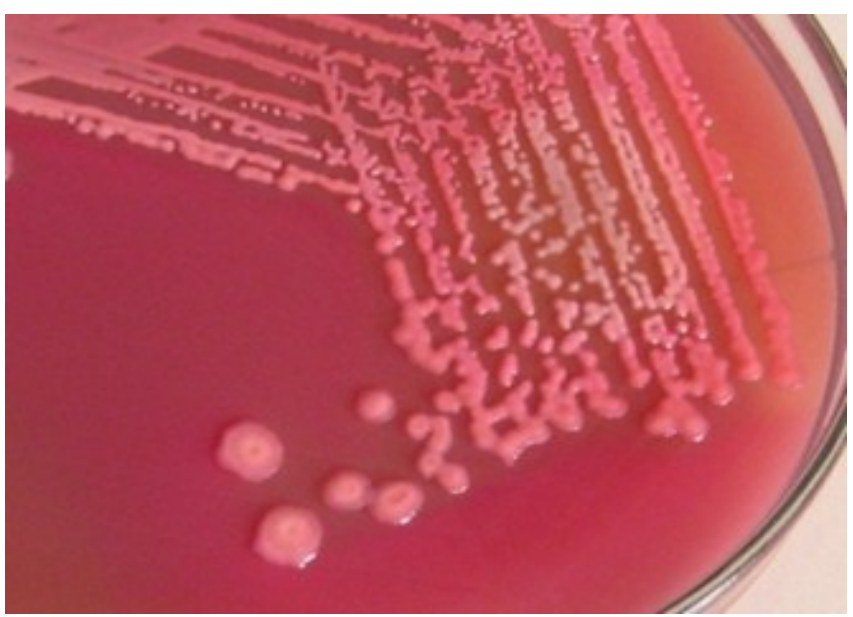

Fig. 4- Lactose Fermenting Colonies on MacConkeys Agar

Antibiotic susceptibility was done by Kirby-Bauer disc diffusion methods and it showed resistance to Amikacin, Gentamicin, Co- 
trimoxazole, Ciprofloxacin, Tobramicin, Cefotaxime, Ampicillin, Ceftazidime and susceptibility to Ceftazidime/clavulanic acid and Imipenem. Antibiotic therapy was administered based on the strain susceptibility e.g. amoxicillin-clavulanate intravenously. The patient improved gradually with favorable outcome.

\section{Discussion}

In the genus Pantoea, $P$. agglomerans is the most commonly isolated species in humans, resulting in soft tissue or bone and joint infections following penetrating trauma by vegetation [5-6]. It is recognized as an agent of endogenous nosocomial infections and able to cause epidemics among hospitalized patients when associated with the use of contaminated intravenous products due to its ability to grow in commercial infusion fluids. A nationwide epidemic of $P$. agglomerans septicemia due to contaminated intravenous products has been reported by Mackel, et al in 1975 [7]. Present patient was not infected from endogenous source and it was not hospital acquired as it was not associated with use of contaminated intravenous products. De Champs, et al in 2000 from France reported $P$. agglomerans in two cases of septic monoarthritis after thorn and wood sliver injuries. P. agglomerans isolated from these two cases were resistant to Amikacin only and were susceptible to rest of antibiotic tested [8]. In this present case we have isolated multidrug resistant $P$. agglomerans and extended spectrum beta lactamases (ESBLs) producer. $P$. agglomerans has phenotypic similarity with Enterobacter species and may be confused with it. But the colony characteristics and growth in liquid broth sausage shaped aggregations or symplasmata will help in differentiation with Enterobacter species. Vitec 2C Systems (bioMerieux, France) definitely helps to do species identification. Diabetes mellitus provides conducive atmosphere as there is associated compromise in immunity.

Milanowski, et al in 2003 and De baere, et al in 2004 have reported occupational respiratory infections and skin allergy due to $P$. agglomerans [9]. Maria Carla Liberto, et al in 2009 reported six cases of sepsis by $P$. agglomerans from Italy teaching hospital [10]. Bennet, et al (1995), Wagner, et al (1994), Matsaniotis, et al (1984) have reported outbreaks due to contaminated intravenous solutions and stored blood products as well as "cotton fever" in intravenous drug abusers [11].

\section{Conclusion}

In last decade sporadic cases due to $P$. agglomerans were reported from USA, Brazil, Spain and Belgium. This report presents probably the first case from India where $P$. agglomerans was the sole pathogen isolated from an infected synovial fluid. We believe that the unnoticed knee injury by road accident and diabetes mellitus increased susceptibility of the infection due to $P$. agglomerans. As $P$. agglomerans is frequently found in water and soil, our report highlight the fact that, this could be a source of infection during accident and is the probable source of infections in our patient. There is a need for microbiologists to identify and report such emerging new members as sole source of causative agent from family Enterobacteriaceae. Physicians and surgeons should be aware of multidrug resistant $P$. agglomerans propensity as a cause of septic arthritis.

\section{References}

[1] Van Rostenberghe H., Noraida R., Zeehaida M., et. al. (2006) Jpn J. Inf. Dis. 59, 120-1.

[2] Cruz A.T., Cazacu A.C., Allen C.H., et. al. (2007) J. Clin. Microbial 45(6), 1989-1992.

[3] Kartz A., Greenberg D., Barki Y., et. al. (2003) Arch. Dis. Child., 88, 542-4.

[4] Habsah H., Zeehaida M., Van Rostenberghe H., et. al. (2005) J. Hosp. Inf. 61, 213-8.

[5] Gavini F., Mergaret J., et al. (1989) Int. J. Syst. Bacterial, 39, 337-45.

[6] Geere J.W., (1977) Can. Med. Assoc. J. 116, 517-519.

[7] Bergman K.A., Arends J.P., Scholvinck E.H., et. al. (2007) Pedi. Inf. Dis. J. 453-4.

[8] De Champs C., Le Seaus S., Dobust JJ., et. al. (2000) J. Clin. Microbiol. 38. 460-1.

[9] Fattauer F.E., Khan M.A. (1978) Arch. Intern. Med. 138-788.

[10]Stromqvist B., Edlund E., Lidgren L. (1985) Acta. Orthop. Scand. 56, 342-3.

[11]Maria C.L., Giovanni M.R. (2009) New Microbiologia. 32(1), 119-123.

[12]Bennett S.N., McNeil M.M., Bland L.A., et. al. (1995) N. Engl. J. Med. 333, 147-54 